ARTICLE

\title{
CpG island-mediated global gene regulatory modes in mouse embryonic stem cells
}

Samuel Beck ${ }^{1}$, Bum-Kyu Lee ${ }^{1}$, Catherine Rhee ${ }^{1}$, Jawon Song ${ }^{2}$, Andrew J. Woo ${ }^{3}$ \& Jonghwan Kim ${ }^{1,4,5}$

Both transcriptional and epigenetic regulations are fundamental for the control of eukaryotic gene expression. Here we perform a compendium analysis of $>200$ large sequencing data sets to elucidate the regulatory logic of global gene expression programs in mouse embryonic stem (ES) cells. We define four major classes of DNA-binding proteins (Core, PRC, MYC and CTCF) based on their target co-occupancy, and discover reciprocal regulation between the MYC and PRC classes for the activity of nearly all genes under the control of the CPG island (CGI)-containing promoters. This CGI-dependent regulatory mode explains the functional segregation between CGI-containing and CGI-less genes during early development. By defining active enhancers based on the co-occupancy of the Core class, we further demonstrate their additive roles in CGI-containing gene expression and cell type-specific roles in CGI-less gene expression. Altogether, our analyses provide novel insights into previously unknown CGI-dependent global gene regulatory modes.

\footnotetext{
${ }^{1}$ Department of Molecular Biosciences, The University of Texas at Austin, Austin, Texas 78712, USA. ${ }^{2}$ Texas Advanced Computing Center, The University of Texas at Austin, Austin, Texas 78758, USA. ${ }^{3}$ School of Medicine and Pharmacology, Royal Perth Hospital Unit, The University of Western Australia, Perth, WA 6000, Australia. ${ }^{4}$ Institute for Cellular and Molecular Biology, The University of Texas at Austin, Austin, Texas 78712, USA. ${ }^{5}$ Center for Systems and Synthetic Biology, The University of Texas at Austin, Austin, Texas 78712, USA. Correspondence and requests for materials should be addressed to J.K. (email: jonghwankim@mail.utexas.edu).
} 
T he precise control of global gene expression programs is governed by multi-layered regulatory steps that include transcriptional $^{1,2}$ and epigenetic ${ }^{3,4}$ regulation. Both processes are mediated by the combinatorial actions of hundreds of trans-acting DNA-binding proteins $(\mathrm{DBPs})^{5}$ and numerous target cis-regulatory elements ${ }^{1}$. Recent advances in highthroughput technologies, such as massive parallel sequencing in combination with chromatin immunoprecipitation (ChIP-seq) and gene expression profiling (RNA-seq), have enabled researchers to identify unbiased genome-wide interactions between DBPs and their genomic target loci, and further enlighten the functional outcomes. Not only the acquisition but also comprehensive analyses of large data sets have become crucial for elucidating the elaborate regulatory mechanisms of global gene expression ${ }^{6}$.

Transcriptional regulation has been suggested as one of the key steps in maintaining the identity of embryonic stem (ES) cells represented by two terms: self-renewal and pluripotency ${ }^{7}$. In addition to the identification of core transcription factors (TFs), such as OCT4, SOX2 and NANOG ${ }^{8-11}$, subsequent studies on the expansion of transcriptional regulatory circuitry have suggested that global gene expression regulation in ES cells is achieved by functionally separable regulatory sub-modules ${ }^{12,13}$. This modular regulation requires close interactions between multiple DBPs including TFs and chromatin regulators and a distinct set of their chromosomal targets. This cooperative modular action not only underscores the importance of studying multiple DBPs within specific regulatory units but also elucidates the significance of the systematic identification of their genomic targets and associated chromatin or DNA modification states.

CpG islands (CGIs) are DNA elements with high GC contents, existing mostly without DNA methylation, and surrounding $>60 \%$ of the transcriptional start sites (TSSs) of eukaryotic genes $^{14,15}$. Early genomic sequencing analysis has shown that CGIs exist invariably on almost all housekeeping genes and less frequently on tissue-specific genes ${ }^{16-18}$. Although CGIs have been previously suggested as one of the important regulatory elements influencing the transcriptional activity of many genes ${ }^{19,20}$, only a few CGI-specific binding DBPs, such as KDM2A, CFP1 and TET1 (refs 21-23), have recently been identified. Therefore, the functional significance of CGI-mediated transcriptional and epigenetic regulations is only beginning to be understood. Comprehensive and systematic approaches to understanding the differences between CGI-containing (CGI +$)$ and CGI-less $(\mathrm{CGI}-)$ promoters in their associated gene expression patterns, global regulatory mechanisms and functional implications have not been clearly demonstrated.

In order to elucidate how DBPs interact with and precisely control the cis-regulatory elements over gene expression programs in ES cells especially within the context of CGIs, we conducted an integrative analysis of publicly available data sets with our new data incorporating the DBP occupancies, histone modification signatures, chromatin accessibility and DNA methylation. The tested DBPs were classified into multiple subgroups based on their target co-occupancy. Strikingly, we found predominant regulation in CGI + promoters by the MYC and polycomb repressive complex (PRC)-related DBPs. This CGIdependent regulatory mode further explains the functional segregation between the CGI-containing and CGI-less genes during early embryonic development. We also defined the active enhancers by the Core class DBPs, and showed that these enhancers regulate cell type-specific gene expression programs in ES cells. In summary, our findings provide novel insights into CGI-dependent global gene regulatory modes in ES cells, characterized by the general regulation of CGI + promoters by the MYC and PRC classes, and the tissue-specific regulation of CGI - genes by enhancer binding Core class DBPs.

\section{Results}

Co-occupancy guided classification of DBPs. Previous studies on ES cells have shown the cooperative regulation of DBPs on their common targets ${ }^{7,13,24}$. In order to obtain further insights into DBP-mediated transcriptional regulation in ES cells, 166 genome-wide DBP occupancy data sets were initially tested (157 publicly available ChIP-seq and 9 newly acquired from the bioChIP-seq ${ }^{24}$; Supplementary Dataset 1 ), and we selected 105 high-quality data sets to classify DBPs based on their target cooccupancy on the genome (Supplementary Dataset 2 and Methods section). As a result, we observed six distinct DBP classes and named them based on the representative factors within each class (Core, PRC, MYC, CTCF, REST and P53; Fig. 1a). The Core, PRC and MYC classes were similar to our prior observations ${ }^{12}$ with additional DBPs, and we identified three new classes (CTCF, REST and P53; Fig. 1a and Supplementary Dataset 2). Notably, the MYC class included the elongation factors ${ }^{25}$ and general TFs, in addition to other previously known $\mathrm{DBPs}^{12,13}$. The CTCF class consisted of insulator protein, CTCF and cohesion complex members. In addition to four major classes (Core, PRC, MYC and CTCF; Fig. 1a), two small classes, P53 and REST, showed unique binding patterns, indicative of their distinct roles in ES cells (Fig. 1a). We noticed that some DBPs shared targets across multiple classes, for instance, TET1 (ref. 22) and KDM2A (ref. 21) shared targets with DBPs in the PRC and MYC classes. The mediators ${ }^{26,27}$ and elongation factors ${ }^{28}$ in the Core class also shared binding sites with the members of the MYC class (Fig. 1a and Supplementary Fig. 1a), implying that these are functionally important in connecting multiple classes of DBPs.

As shown in Supplementary Fig. 1b,c, we observed a positive correlation between the co-occupancy and the strength of the DBP binding only within the same class of DBPs, suggesting that DBPs from the same class function cooperatively. As previously

Figure 1 | Co-occupancy guided identification of DBP classes and their associated chromatin status. (a) Co-occupancy guided classifications of DBPs. Heatmap shows six distinctive clusters (colour-coded bars) from an unsupervised hierarchical clustering of co-occupancies for 105 DBPs. Representative DBPs and their putative functions are shown below. The extent of co-occupancy between two DBPs was monitored with the deviation of actual cooccupancy from randomized expectation (Z-score, see Methods section). Red: Core class (19 DBPs, 29 experiments), blue: PRC class (7 DBPs, 21 experiments), green: MYC class (18 DBPs, 22 experiments), black: CTCF class (6 DBPs, 21 experiments), cyan: P53 class (single DBP, 2 experiments) and yellow: REST class (single DBP, 4 experiments). (b) Distance distribution of DBP-binding sites from transcriptional start sites (TSSs). DBPs are listed in the same order as in Fig. 1a. Note that DBP-binding sites in the PRC and MYC classes are generally enriched nearby TSSs, while the binding sites of the Core and CTCF classes are further away from TSSs. (c) Association of each DBP class with a unique chromatin status. Each colour-coded bar graph, excluding me-CpG, shows the portion containing a specific histone mark or DNase I hypersensitivity ( $y$ axis) within the given DBP-binding sites ( $x$ axis, same order as in Fig. 1a). Me-CpG (grey) indicates the average portion ( $y$ axis) of CpG methylation within the given DBP-binding sites ( $x$ axis). Genome-wide average portion of CpG methylation (0.6474) is shown with a grey dashed line. (d) Association of increased co-occupancy of DBPs within the class with specific histone marks. The portions containing chromatin marks or percentage of $\mathrm{CpG}$ methylation ( $y$ axis) within the centre (300 bp) of the region co-occupied by indicated number of DBPs ( $x$ axis) are plotted with different colour as indicated in Fig. 1c. 
a

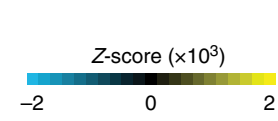

b

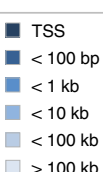

$0 \%$

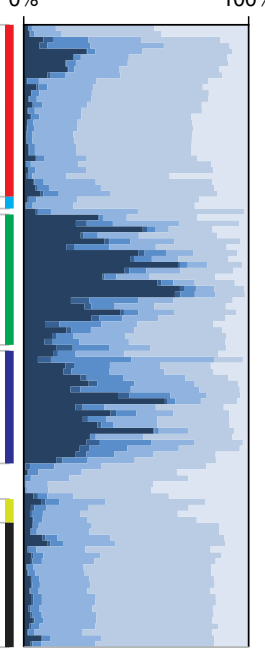

Tட Elongation factor (ELL2, AFF4)

Core class

- Core TF: OCT4, NANOG, SOX2

- Other TF: ESRRB, PRDM14, SMAD3,

DAX1, NR5A2, TCF3, BRD

- Mediator: MED1, MED12

- Elongation factor: ELL2, AFF4

- Condensine protein: $\mathrm{CAPH} 2$

- Histone deacetylase: HDAC1,2, CHD4

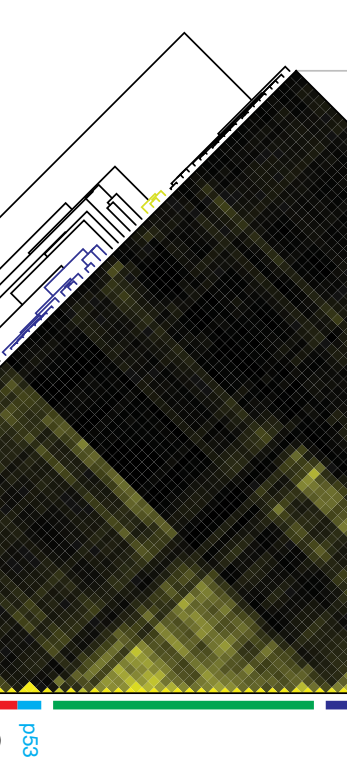

MYC \& associated protein: MYC NMYC, MAX, TIP60, DMAP1, ZFX, MOF, KLF4, E2F1, E2F4

- General TF: TBP, TAF1, TAF3

Elongation factor: NELFA, SPT

Condensine protein: CAPD3
H3K4 methyltransferase: DPY30

c

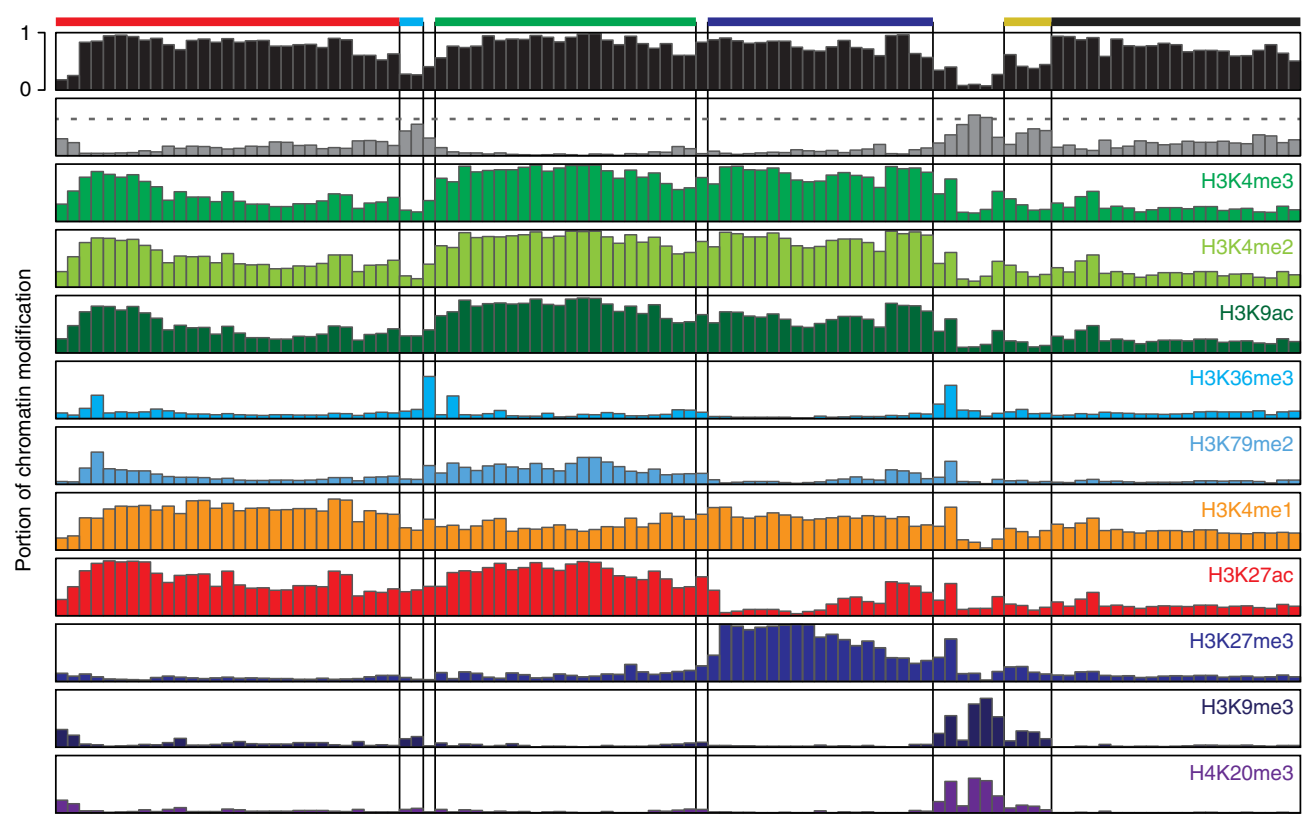

PRC class PRC1: RING1B PRC2: EZH2, SUZ12,

JARID2, PCL2

PRC/MYC linker: TET1, KDM2A

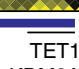

KDM2A

- CTCF

Cohesin complex: SMC1,3

STAG1,2, RAD21
Accessibility (DNase I)

Silent region

(Me-CpG)

Promoter

Active gene body

Enhancer

Activation

Repression

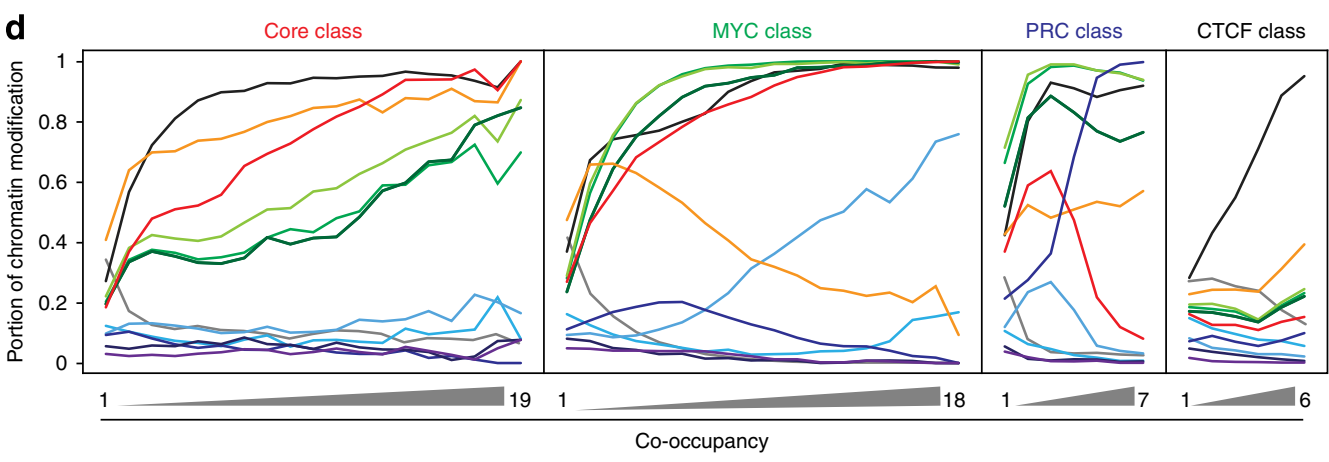


reported, the PRC and MYC classes generally occupy sites near TSSs, whereas the Core and CTCF classes generally localize further away from the TSSs, suggesting a unique proximal or distal regulatory mode for each class (Fig. 1b). Since the target occupancy of DBPs is often associated with specific chromatin marks ${ }^{29-31}$, we examined the association between the cooccupancy of DBPs and 10 histone modification signatures, chromatin accessibility (DNase I hypersensitivity regions, DHRs) or DNA methylation (Supplementary Dataset 3 and Fig. 1c,d). The Core class targets were highly enriched in DHRs with a putative enhancer histone signature, H3K4me1 (ref. 31). The MYC class preferentially bound to DHRs with the active histone markers, $\mathrm{H} 3 \mathrm{~K} 4 \mathrm{me} 3$ and $\mathrm{H} 3 \mathrm{~K} 27 \mathrm{ac}$. The binding regions of the PRC class harboured both active $(\mathrm{H} 3 \mathrm{~K} 4 \mathrm{me} 3)$ and repressive (H3K27me3) markers as previously reported ${ }^{19,29}$. Interestingly, we found that the PRC class binding regions are mainly within DHRs, implying that unlike in previous reports ${ }^{32,33}$, chromatin condensation is not an absolute requirement for PRC-mediated gene repression. The binding sites of all four major classes are enriched within the DHRs with the depletion of DNA methylation (Fig. 1c,d). Taken together, our results show that DBPs within the same class share many common regulatory features and that each class may have a distinct role in global gene regulation.

The MYC and PRC classes predominantly occupy CGI+ promoters. As shown in Fig. 1a, our analysis revealed that previously reported CGI-binding proteins, such as KDM2A (ref. 21) and TET1 (ref. 22), share targets with the MYC and PRC classes. In addition to a prior report that suggested the CGIdependent regulation of $\mathrm{PRC1/2}$ (ref. 29), our data imply that CGIs may be equipped to control local gene activity in combination with specific DBPs ${ }^{15}$. Since the MYC and PRC classes tend to occupy proximal promoter regions among the multiple DBP classes we defined (Fig. 1a,b), we hypothesized that the major roles of the MYC and PRC classes are restricted to CGI + promoters. To test this hypothesis, we examined the extent of the global target occupancy of DBPs in all classes on the CGI + and CGI - promoters (Fig. 2a). Markedly, most DBPs in the MYC and PRC classes showed a strong occupancy on the CGI + promoters only (Fig. 2a and Supplementary Fig. 2). Subsequent tests for global gene expression showed that the gene activity under the control of the CGI + promoters (hereafter, $\mathrm{CGI}+$ genes) is significantly higher than the activity of genes with CGI - promoters (CGI - genes; Fig. 2b). Further testing of the gene expression profiles from 91 tissue samples ${ }^{34}$ revealed that the overall levels of the CGI + genes are much higher than the levels of the CGI - genes (Fig. 2c). These results suggest a general 'on' state of the CGI + genes in ES cells. The expression of the CGI - genes, however, was skewed to a minimum value in most samples, while being selectively active in a few samples, indicating a general 'silent' and a context-dependent 'on' state of the CGI - genes. Taken together, these findings strongly suggest two CGI-dependent modes of global gene regulation: one for $\mathrm{CGI}+$ genes and the other for CGI - genes. a

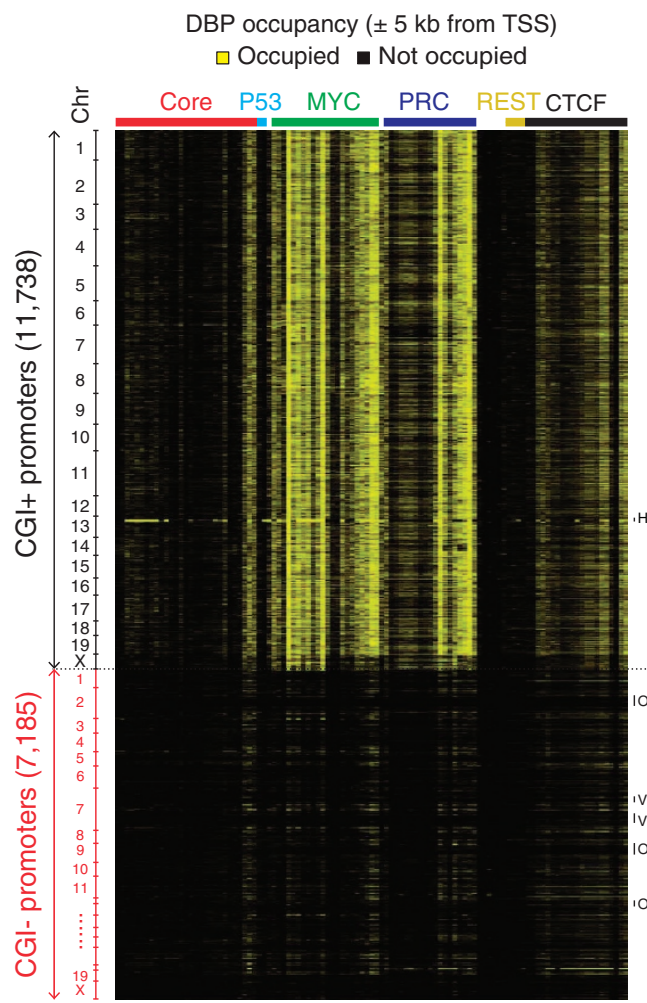

b
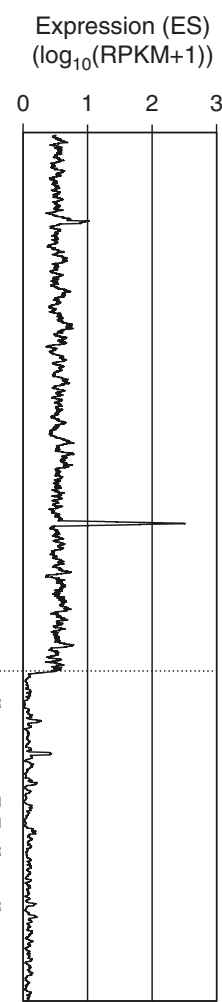

C

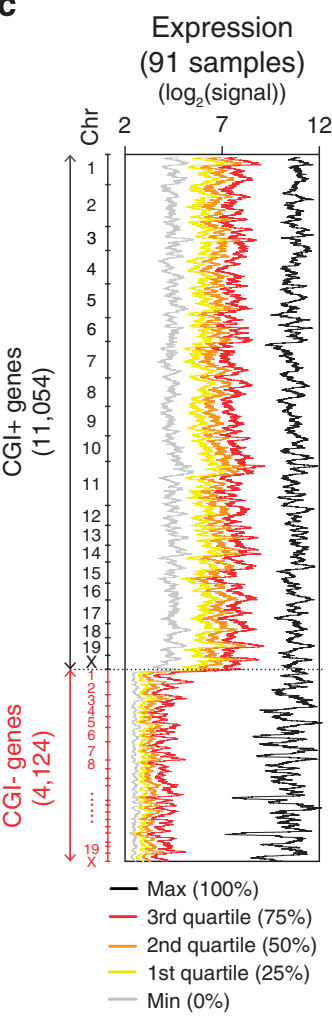

Figure 2 | Predominant regulation of the MYC and PRC classes on CGI + promoters. (a) Heatmap presenting DBP occupancies (same order as in Fig. 1a) within $\pm 5 \mathrm{~kb}$ from TSSs of CGI + and CGI - genes (vertical, chromosomal order). Gene clusters showing distinct binding patterns are shown on the right as follows. HS, histone gene cluster; OR, olfactory receptor gene cluster; VM, vimentin gene cluster. (b) The expression value of each gene in ES cells is plotted using a moving average (window size: 64, bin size: 1; genes are in chromosomal order as in Fig. 1a) across CGI + and CGI - genes. Expression levels are shown in $\log _{10}($ RPKM +1$)$ scale. RPKM, reads per kilobase per million. (c) Moving average plots (window size: 64, bin size: 1 ; genes are in chromosomal order) showing global gene expressions of 91 different tissues or cell lines (http://biogps.gnf.org) ${ }^{34}$. Expression values are ranked for each gene, and maximum, quartiles and minimum values among samples are plotted with different colours as indicated. 
The MYC and PRC classes determine the activity of CGI+ genes. We found that up to $99.4 \%(11,667$ out of 11,738$)$ of all CGI + genes are occupied by at least one factor from either the MYC or PRC class, while $<10 \%$ of the CGI - genes are their targets (Fig. 3a and Supplementary Fig. 3a). As expected, the activity of the CGI + genes showed a positive correlation with MYC class co-occupancy and a negative correlation with the PRC class (Fig. 3a,b). In accordance with this observation, the active $\mathrm{CGI}+$ genes harboured high levels of MYC-associated active histone markers, such as $\mathrm{H} 3 \mathrm{~K} 4 \mathrm{me} 3$ and $\mathrm{H} 3 \mathrm{~K} 27 \mathrm{ac}$, whereas the inactive $\mathrm{CGI}+$ promoters harboured repressive $\mathrm{H} 3 \mathrm{~K} 27 \mathrm{me} 3$ markers (Fig. 3a,c, see also Fig. 1c,d and Supplementary Fig. 4a). Only a small number of highly active CGI - genes showed a marginal level of MYC class binding with active histone markers (Fig. 3a and Supplementary Fig. 4a). Collectively, these results suggest that there is selective regulation of CGI + genes by the MYC and PRC classes. In agreement with prior reports of non-linear target gene amplification ${ }^{35,36}$ and RNA polymerase II (Pol II) release of $\mathrm{Myc}^{25}$ as well as the direct inhibition of target gene expression by disassembling the Pol II pre-initiation complex by $\mathrm{PRC}^{37}$, our analyses indicate that the activity of nearly all CGI + genes can be generally explained by the reciprocal regulation between the MYC and PRC classes (Fig. 3b).

Functional separation between the CGI + and CGI - genes. Our analyses revealed that there are marked differences in global gene activity, DBP occupancy and histone signatures between CGI + and CGI - genes (Figs 2 and 3). Although more tissuespecific activity of CGI - genes was previously suggested ${ }^{17,38}$, the functional segregation between CGI + and CGI - genes has not been systematically addressed. To address this issue, we looked into gene expression variations across different tissue samples ${ }^{34}$

a
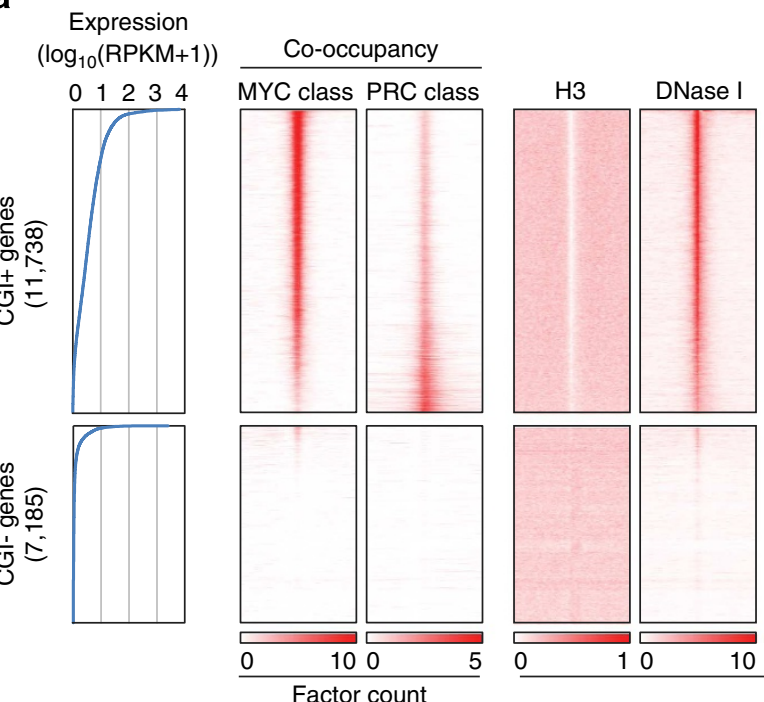

H3K4me3 H3K27me3 H3K4me1 H3K27ac Pol II
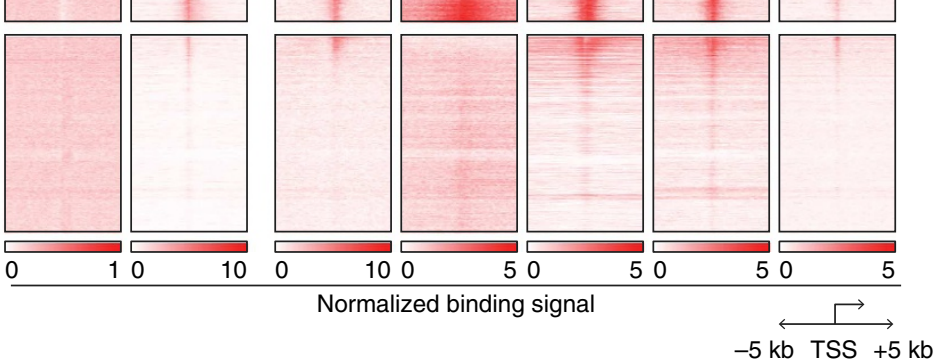

b

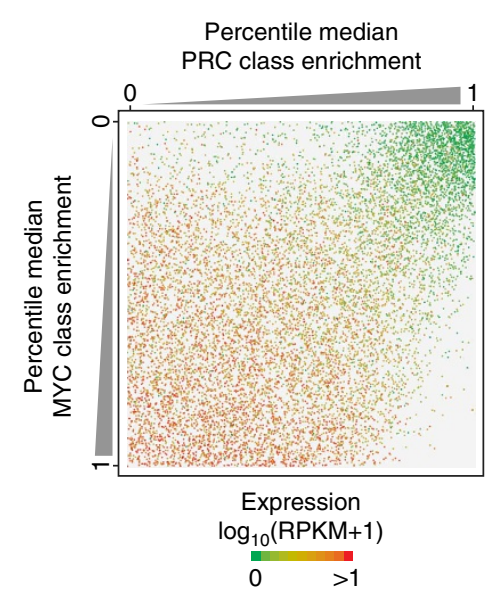

C

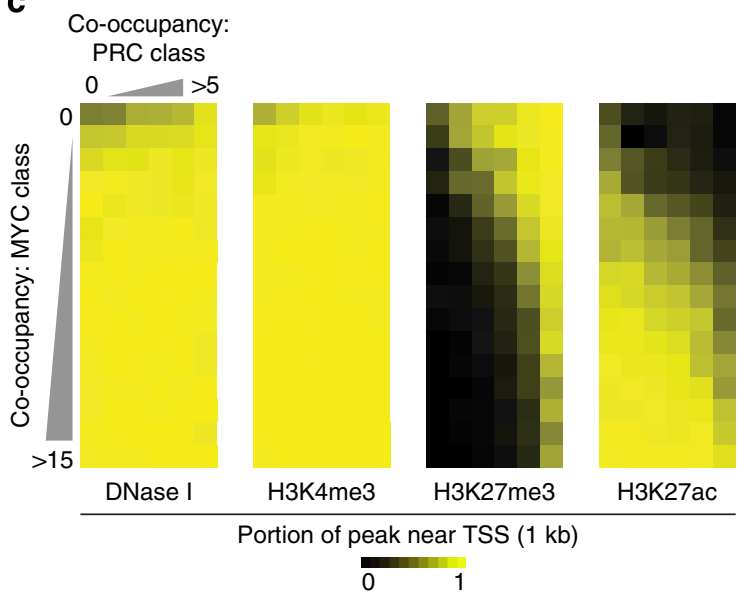

Figure 3 | The MYC and PRC classes reciprocally determine the activity of CGI + genes. (a) Profiles of the MYC and PRC class co-occupancies, histone marks, DNase I hypersensitivity signal and Pol II occupancy within $\pm 5 \mathrm{~kb}$ from the TSSs of CGI + and CGI - genes. All protein-coding genes are sorted by their expression values (left panel, blue lines) and ChIP-seq profiles are shown as heatmaps. (b) A dot plot showing the correlation between the combinatorial occupancy of PRC ( $x$ axis) and MYC ( $y$ axis) class DBPs and corresponding CGI + gene expression. Each dot indicates an individual gene. Total 11,738 genes were shown (see Methods section for detail). (c) Heatmaps showing the MYC and PRC class co-occupancy and their associated chromatin status near the TSSs of CGI + genes. Each cell in the heatmaps indicates the average portion of the indicated chromatin mark within $1 \mathrm{~kb}$ regions of the TSSs ( \pm 500 bp from TSS) co-occupied by the indicated number of DBPs from the MYC (vertical) and PRC (horizontal) classes. 
by performing two independent hierarchical clustering analyses of the CGI + and CGI - genes (Fig. 4a,b; see Methods section). Among the CGI + genes, large groups of gene clusters (gene sets; numbers in black) were co-regulated and synchronously activated in the tissues from the same developmental origins (Fig. 4a). In contrast, the clustering of the CGI - genes generated smaller gene clusters (numbers in red) within a limited number of tissues (Fig. 4b).

We then performed gene set overlap tests (see Methods section) to understand the functional implications of the CGI context in developmental stages and tissue specificity, by comparing the gene sets defined from the clustering analyses (Fig. 4a,b) with previously identified active gene sets during development (Gene Expression Database) ${ }^{39}$. The comparison revealed that the gene sets acquired from the CGI + genes are frequently activated throughout early embryonic development, whereas the gene sets from the CGI - genes mostly remained silent (Fig. 4c). Likewise, the comparison of the gene clusters with the previously known mutant phenotypes (Mouse Genome Informatics, MGI ${ }^{40}$ showed that the loss of CGI + genes often a

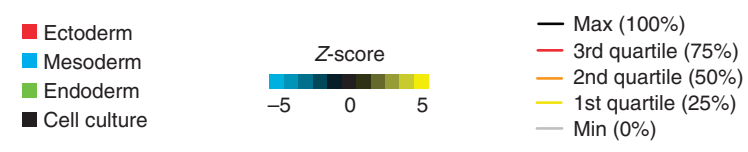

C
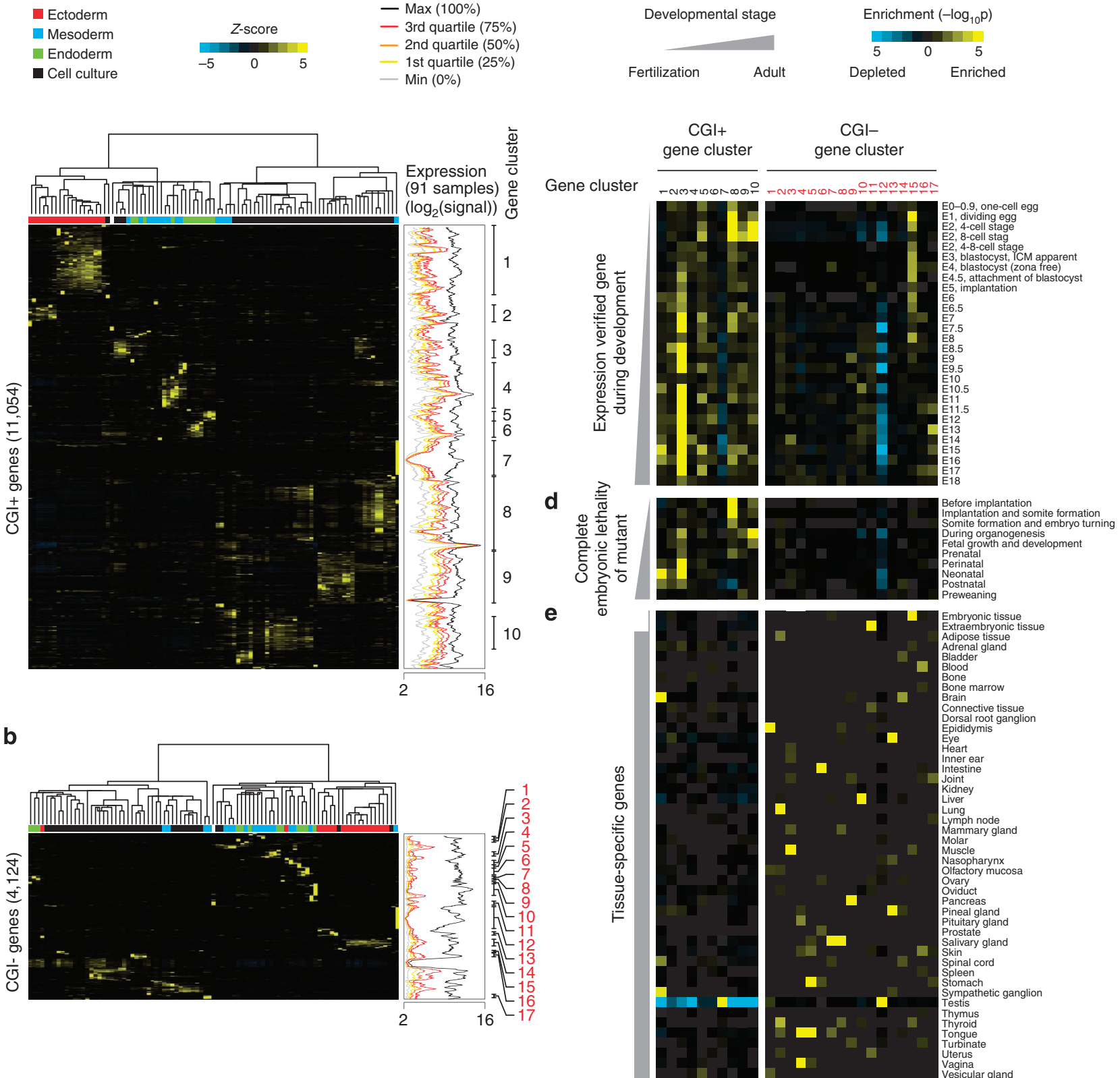

b

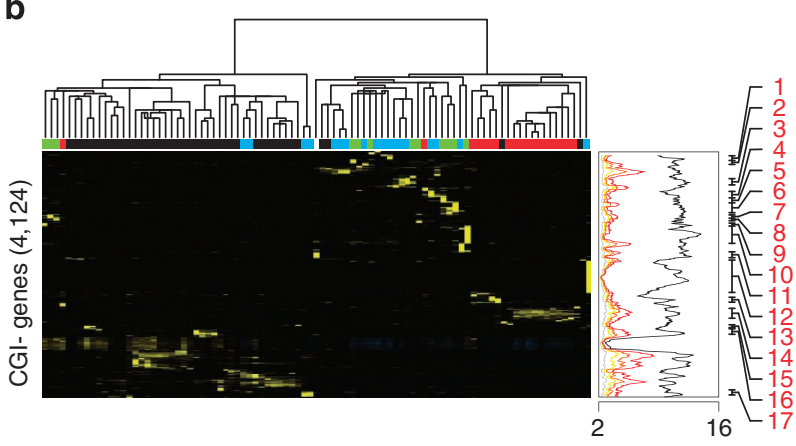

\section{$\mathrm{CGI}+$}

CGI-

Gene cluster - -

Figure 4 | Functional segregation between CGI + and CGI - genes during early embryo development. (a,b) Unsupervised hierarchical clustering of gene expression variances (Z-scores) for CGI + (a) and CGI - genes (b) from 91 tissues or cell lines (http://biogps.gnf.org34; left clustering result). For each sample, the putative origin of embryonic germ layer is indicated with a different colour (upper part of heatmap). Moving average plots in the middle (window size: 64, bin size: 1; genes are ordered as in gene cluster results) show global gene expressions of 91 different tissues or cell lines as shown in Fig. 2c. Gene clusters (gene sets) showing a similar pattern of expression variances are defined, and indicated as numbers in black and red (CGI + and $\mathrm{CGI}$ - genes, respectively; right end). (c-e) Heatmaps showing hypergeometric $P$ values calculated by gene set overlap analyses between gene sets from hierarchical clustering (Fig. 4a,b) and expression verified gene sets during embryo development (c), gene sets showing complete embryonic lethality upon deletion (d) or tissue-specific genes (e) (see Methods section for detail). 
led to embryonic lethality (Fig. 4d). However, the comparison with the tissue-specific gene lists in the adult mouse (Unigene) showed that tissue-specific expression is more distinctive in CGI - genes (Fig. 4e). Collectively, our integrative analysis provides clear evidence showing that there are functionally separated CGI-dependent regulatory modes (Figs 2 and 3). Many active CGI + genes are required for general cellular processes with a basal level of gene activity, whereas the mostly silent CGI - genes are selectively activated in more specialized cell types or tissues.

The Core class selectively defines active enhancers. Among various strategies for identifying enhancers, co-occupancy mapping of a cluster of TFs has been suggested as the most reliable method with fewer false positives and negatives ${ }^{41}$. Our own analyses also showed that DBPs in the Core class occupy distal regulatory regions with a high level of $\mathrm{H} 3 \mathrm{~K} 4 \mathrm{mel}$, the marker used for the chromatin signature of enhancers (Fig. 1c,d). Since the Core class DBPs share many common targets with the factors known to occupy enhancer elements including mediators (MED1 and MED12) as well as co-activators (P300, LSD1 and CHD7) (Fig. 1a and Supplementary Fig. 1a) ${ }^{27,42}$, we sought to define the enhancers using the co-occupancy of the Core class DBPs in ES cells.

We mapped the enhancers along with a number of the cooccupied Core class TFs. To determine the appropriate threshold for mapping reliable enhancers, we compared P300 signals, a representative enhancer mark, from the enhancers we defined based on the co-occupancy of the Core class DBPs and the enhancers defined by other enhancer annotation methods ${ }^{43-47}$. The regions co-occupied by six or more DBPs of the Core class showed more than twofold higher P300 signals than any of the other enhancers defined by the prior methods ${ }^{43-47}$ (Supplementary Fig. 5a), indicating that our enhancer-defining method outperforms the other widely used methods. Using the co-occupancy criterion of at least six DBPs, we defined 8,726 putative enhancers (Fig. 5a and Supplementary Dataset 4, see also Methods section) spanning an average of $1.6 \mathrm{~kb}$ long, and preferentially localizing to distal regions (median $25.9 \mathrm{~kb}$ from TSS). The majority of the enhancers we defined are within DNase I-hypersensitive regions (Fig. 5a), while a large portion of the enhancers defined by the other methods reside within nonaccessible regions, which is indicative of false positives or inactive enhancers (Supplementary Fig. 5b). Similar to recent observations ${ }^{26}$, we found some enhancers spanning up to longer than $5 \mathrm{~kb}$ (253 enhancers; Fig. 5b). We observed a positive correlation between the co-occupancy of the Core class and the activity of their associated genes (hereafter, enhancer target genes; Fig. 5c) or the binding signal of the co-activators or mediators (Fig. 5d).

Recent reports suggested that $\mathrm{H} 3 \mathrm{~K} 27 \mathrm{me} 3$ can serve as a marker for 'poised' enhancers ${ }^{41,43}$. Interestingly, we found that the H3K27me3 signature is almost completely depleted in the enhancers defined by the Core class. Since they still harboured both H3K4me1 and H3K27ac markers (Figs 1d and 5a), these findings suggest that the DBPs in the Core class exclusively occupy active enhancers, but not the poised enhancers in ES cells.

Although the Core class factors generally occupy distal cisregulatory elements (Fig. 1b), some of the enhancers we defined reside near proximal promoters (Fig. 5e), and we found 376 genes associated with multiple enhancers: proximal (within $1 \mathrm{~kb}$ of TSS) and distal (within $20 \mathrm{~kb}$ of TSS) enhancers (Supplementary Dataset 5). Interestingly, these genes are more active than other genes solely associated with proximal enhancers, distal enhancers, super-enhancers ${ }^{26}$ or regulatory elements mapped by chromatin interaction analysis with paired-end tagging ${ }^{48}$ (Fig. 5f). Notably, these multiple enhancer-associated genes include many ES cell-specific regulators, such as Oct4, Nanog, Sox2 and Lin28a (Supplementary Fig. 6a), and the loss of these genes preferentially leads to early developmental failure (Supplementary Fig. 6b), indicating that multiple enhancer-associated genes play important roles in controlling the identity of ES cells.

Enhancer binding regulators are critical for CGI - genes. We showed that the reciprocal regulation between the MYC and PRC classes is predominant in CGI + genes and controls the general activity of almost all CGI + genes (Fig. 3). Since enhancers have been suggested as a critical regulatory component driving cell type- or tissue-specific gene expression ${ }^{46,49}$, we sought to elucidate the general roles of enhancers in CGI-dependent global gene regulation. As shown in Fig. 6a, the co-occupancy of the MYC and PRC classes showed strong positive and negative correlations, respectively, with the activity of only CGI + genes. However, the co-occupancy of the Core class DBPs showed a similar positive correlation with the activities of the CGI + and CGI - genes, indicating that the enhancers defined by the Core class are responsible for the activity of the CGI + and CGI - target genes. Since CGI + genes are generally active in various cell types (Fig. 2c), these results signify that enhancers play additive roles in the activity of their CGI + target genes, but more decisive roles in the activity of CGI - target genes.

In order to further delineate the functional significance of enhancers in the regulation of CGI - genes, we examined the activity of enhancer-associated genes and targets of OCT4, a representative enhancer binding master regulator from the Core class, upon knockout of Oct4 (Fig. 6b and Supplementary Fig. 7). The activities of the CGI + and CGI - genes associated with the enhancers (upper) and OCT4 (bottom) were reduced upon two different perturbations, suggesting that enhancers (or OCT4) regulate both CGI + and CGI - target genes. However, the extent of the expression decrease was significantly larger in the CGI - targets, confirming the more specific roles of enhancers in the CGI - target genes. We further examined the effects of enhancer binding master regulators on their targets in other cell types ${ }^{46}$ : FOXA2 in liver cells, PU.1 in B cells and MYOD in C2C12 cells (Fig. 6c). For both CGI+ and CGI - genes, the overall activity of the genes associated with the master regulator was greater than the activity of the genes not associated with the master regulator. However, we consistently observed significantly more activity from the CGI genes regulated by master regulators, whereas the non-target CGI - genes showed almost no detectable activity (Fig. 6c). Since CGI + genes are generally active throughout various cell types (Fig. 2c), our results not only indicate that enhancers synergistically increase the activity of CGI + target genes but also illustrate that enhancer-mediated transcriptional regulation is more critical for the activity of CGI - target genes. Consistent with this, ectopic expression of $M y o D$ in fibroblasts led to significant global induction of the CGI - target genes, whereas the CGI + target genes did not show a significantly unified response (Fig. 6d).

We further examined the activity of CGI + and CGI - genes in ES versus liver cells. For CGI + genes, the expression profiles of the ES and liver cells showed a strong positive correlation, indicating that the activity of many CGI + genes is similar in both cell types. Although mildly skewed towards their tissue of origin, CGI + genes that are targets of tissue-specific enhancer binding proteins (OCT4 for ES and FOXA2 for liver cells) also 
a

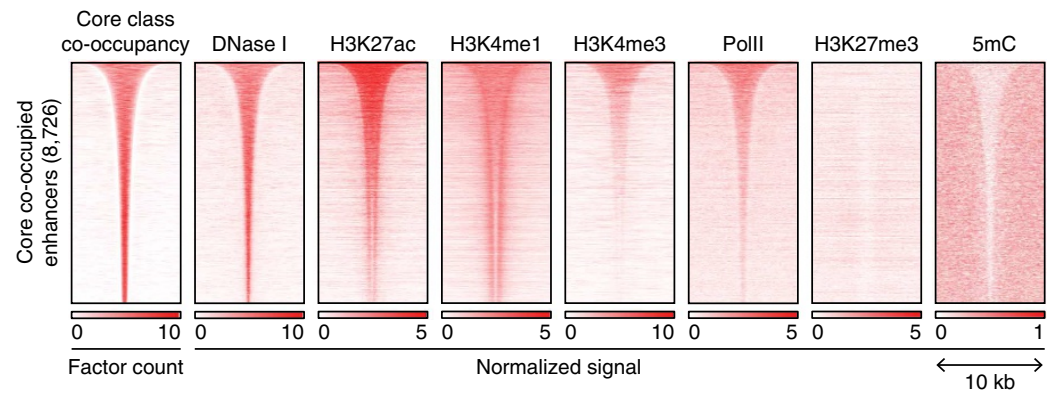

b

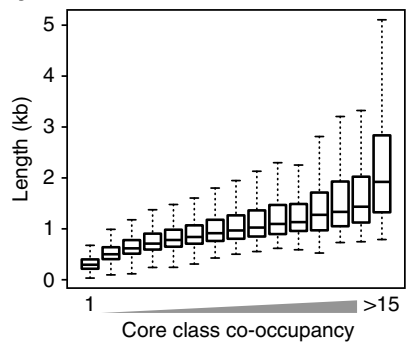

C

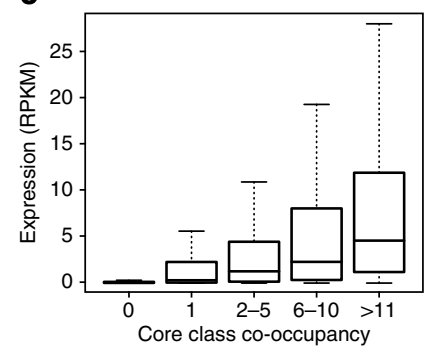

d

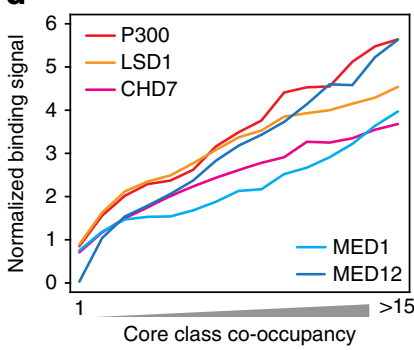

e

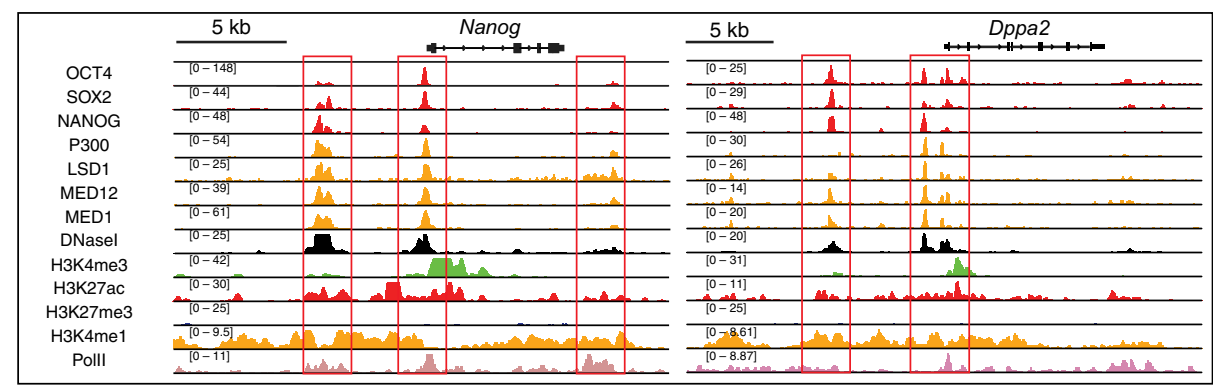

f

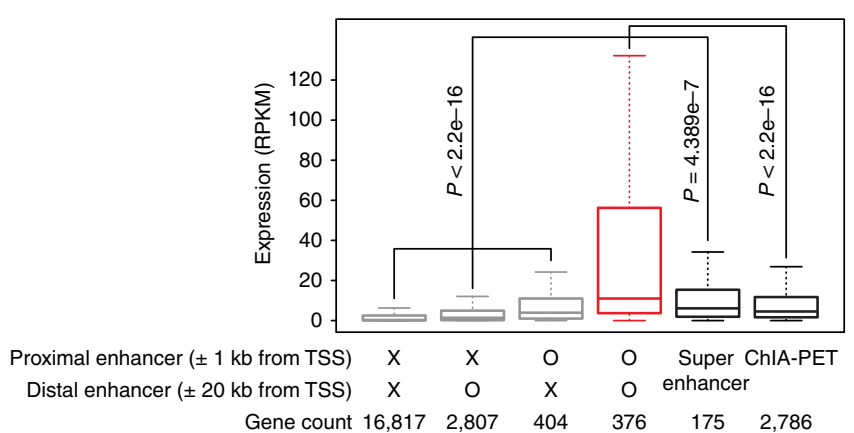

Figure 5 | The Core class DBPs define active enhancers in ES cells. (a) Profiles showing the co-occupancy of the Core class DBPs, chromatin accessibility (DNase I), Pol II occupancy, histone modification marks and DNA methylation within $\pm 5 \mathrm{~kb}$ from the centre of the Core class co-occupied ( $>5$ DBPs; Supplementary Dataset 4) enhancers. The enhancers are sorted by the lengths of their co-occupied regions. (b-d) The correlation between the cooccupancy of the Core class ( $x$ axis) and the length distribution of the regions co-occupied by the Core class DBPs (b), target gene activity ( \pm 5 kb) (c), and normalized binding signals of co-activators (P300, LSD1 and CHD7) and mediators (MED1 and MED12) (d). (e) Examples of genes controlled by multiple enhancers. ChIP-seq signal tracks for the Core class DBPs (OCT4, SOX2 and NANOG), co-activators (P300 and LSD1), mediators (MED1 and MED12), Pol II, open chromatin (DNase I) and diverse histone marks are shown. Enhancer elements are highlighted with red rectangular boxes. (f) Box plot showing the expression of genes regulated by different types of enhancers; proximal ( $\pm 1 \mathrm{~kb}$ from TSS), distal ( $\pm 20 \mathrm{~kb}$ from TSS) and multiple enhancers (both proximal and distal). ' $O$ ' and ' $X$ ' indicate the presence and absence of enhancers in surrounding regions of TSSs, respectively. Expressions of genes regulated by super enhancers ${ }^{26}$ and genes physically interacting with enhancers identified by chromatin interaction analysis with paired-end tagging $(\mathrm{ChIA-PET})^{48}$ are also shown. $P$ values were calculated from Wilcoxon signed-rank test between two groups.

showed a similar expression pattern (Fig. 6e, left panel). For the CGI - genes, in contrast, the overall distribution of the gene expression profile was highly biased towards each axis, demonstrating the tissue-specific nature of the gene activity. Moreover, the CGI - targets of OCT4 or FOXA2 showed strong tissue-biased gene expression patterns (Fig. 6e, right panel). Taken together, our analysis strongly indicates that the enhancer binding proteins play roles in determining tissue-specific gene expression programs for the CGI - target genes, while these proteins play additive roles for the CGI + target genes. 

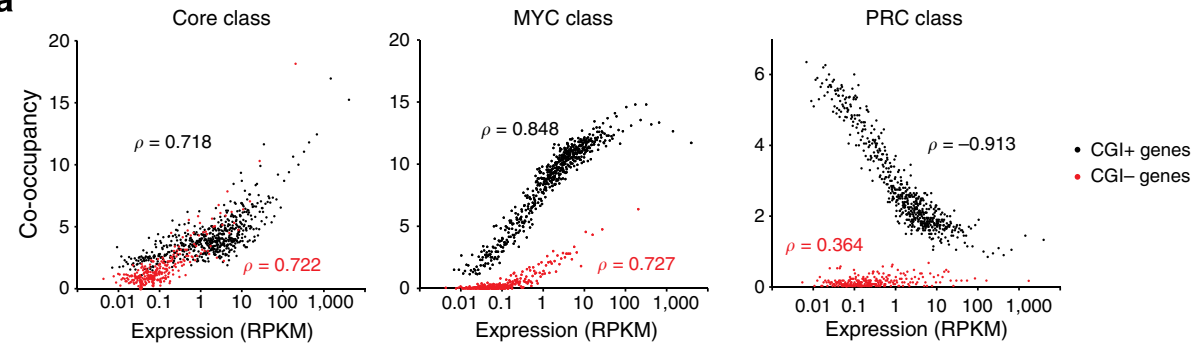

b
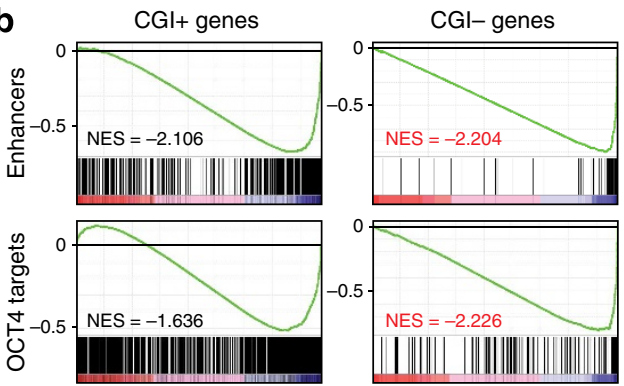

Expression after Oct4 KO (2d) Increased Decreased

C
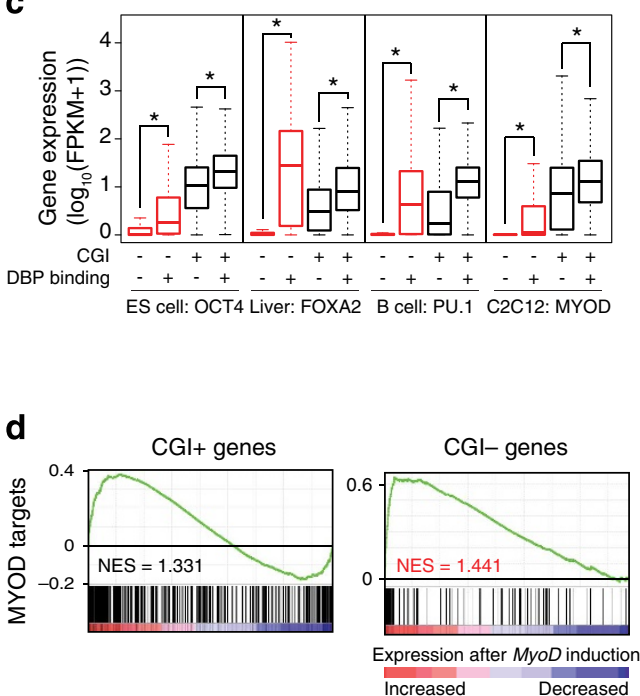

e

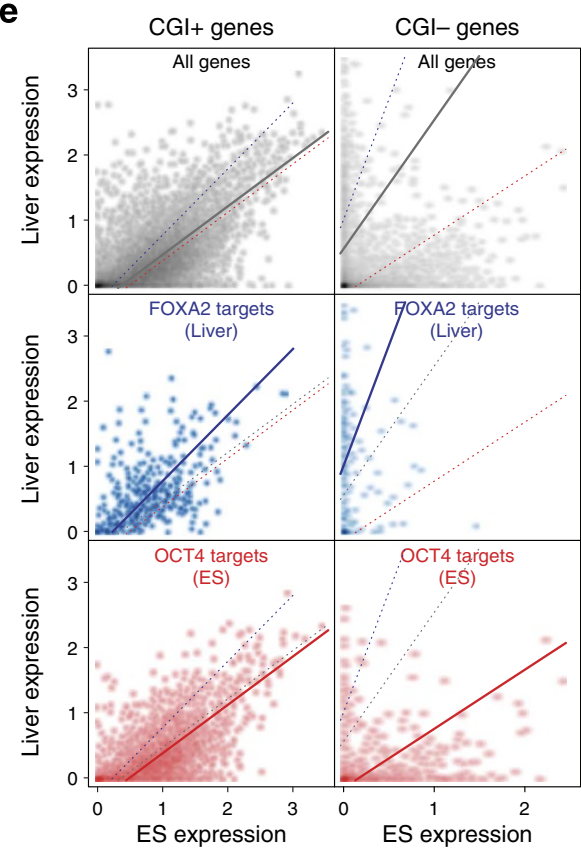

f

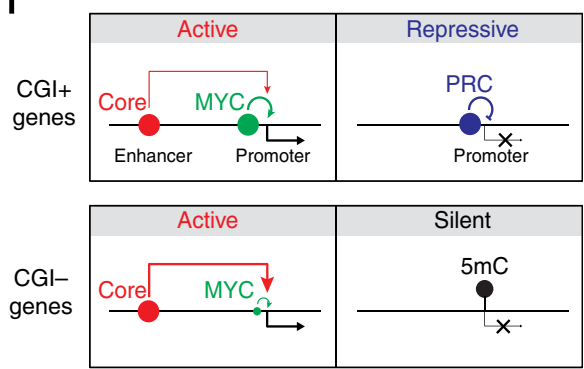

Figure 6 | Enhancer-binding master regulators control the activity of tissue-specific CGI - genes in addition to general activity of CGI + genes.

(a) Correlations between the co-occupancy of DBPs in each class and the expression of CGI + (black dots) or CGI - (red) target genes. Genes were sorted with expression levels and binned into every 20 genes. Each dot represents an average expression and co-occupancy of a group of genes. Gene groups with an average RPKM >0.01 are shown. $\rho$ indicates Spearman correlation coefficient. (b) Gene set enrichment analyses (GSEA) using gene expression profile from conditional Oct4 KO ES cells (GSE10477; ref. 62). Gene sets for enhancers (Fig. 5a) and OCT4 targets ( \pm 5 kb from TSS, GSM288354; ref. 13) were used. NES (normalized enrichment score). (c) Box plots showing the effect of tissue-specific enhancer-binding master regulators on their CGI + (black boxes) or $\mathrm{CGI}-$ (red) targets. Specific cell types and tissue-specific master regulators are indicated. Target genes were assigned within $\pm 5 \mathrm{~kb}$ from the centre of the binding sites. FPKM, fragments per kilobase per million. ${ }^{\star} \rho<2.2 \mathrm{e}-16$ (Wilcoxon signed-rank test). (d) GSEA using gene expression profile from MyoD induction in MEF cells (GSE6487) ${ }^{64}$ and MYOD targets (GSE36024) ${ }^{59}$. (e) Enhancer target gene expression (CGI + or CGI - genes) between ES cells and liver cells. Expression of all genes (grey dots), FOXA2 targets (blue) and OCT4 targets (red) are shown in two-dimensional dot plots. Coloured trend lines are from least square linear regressions of each test. Gene expressions are shown as $\log _{10}(\mathrm{FPKM}+1)$. (f) A model of CGI-mediated global gene regulatory modes in ES cells. The MYC and PRC classes reciprocally regulate CGI + genes, whereas the Core class regulates both CGI + and CGI - target genes. A majority of CGI - genes are silent with DNA methylation while only a small subset of them are activated by the Core and MYC classes.

\section{Discussion}

We performed a compendium analysis, integrating various DBP occupancies and chromatin status data sets, to elucidate global regulatory mechanisms in the context of CGI. After mapping multiple DBP classes based on co-occupancy, we revealed predominant reciprocal regulation between the MYC and PRC classes on CGI + genes (Figs 2 and 3). We also showed the roles of the ES cell-specific enhancers defined by the Core class on 
CGI + and CGI - genes, revealing the CGI-dependent modes of global gene regulation in ES cells and other cell types (Fig. 6f).

Unlike previous reports ${ }^{19,30}$, we found that a large portion of the CGI + promoters remained bivalent even in differentiated tissues (Supplementary Fig. 3b), suggesting that the reciprocal regulation of CGI + genes by the MYC and PRC classes may be a common feature shared in other cellular contexts. Although many inactive $\mathrm{CGI}+$ promoters harbour $\mathrm{H} 3 \mathrm{~K} 27 \mathrm{me} 3$ markers, the majority of the CGI - genes are silenced without a H3K27me3 marker in the ES cells and other differentiated tissues that we tested (Supplementary Fig. 4a,b). This 'by-default silent' state of the CGI - genes may allow the efficient management of limited resources in the cells, since most of these genes are not abundantly expressed or critical during the early developmental stages (Fig. 4c,d). This observation also indicates the necessity of the PRC-independent repression mechanism for the CGIpromoters. The unique mode of gene silencing on CGIpromoters possibly mediated by methyl-DNA-binding proteins will be of great interest for future studies (Supplementary Fig. 4c).

Consistent with previous reports showing the enrichment of tissue-specific DBP recognition motifs in the distal regulatory elements of CGI - genes ${ }^{38}$, we showed that the enhancer binding proteins govern tissue-specific CGI - gene expression programs. This is also consistent with the recent report of 'super-enhancers' occupied by master regulators that control cell type-specific gene expression $^{26}$. The super-enhancers reported in ES cells, in turn, fall into the subset of enhancers we defined in this study (Supplementary Fig. 5c). Since tissue-specific CGI- gene expression programs turn on in parallel with terminal differentiation (Fig. 4e), further understanding of tissue-specific gene regulation mediated by enhancer binding master regulators, particularly in CGI - genes, would be helpful for developing more direct methods of controlling cell fates through induced trans-differentiation or direct reprogramming.

Taken together, our compendium analyses provide a conceptually unique perspective in understanding the global gene regulatory mechanisms. We used DBPs to define regulatory classes based on their target co-occupancy. Such defined classes then serve as powerful analytical tools in interrogating the global gene regulatory modes in ES cells with additional large data sets. Our analyses reiterate a unifying view of global transcriptional and epigenetic regulatory modes, especially incorporating CGIs as a crucial regulatory portal in determining general or tissue typespecific gene expression programs.

\section{Methods \\ Cell cultures. Mouse J1 ES cell lines were maintained as described previously ${ }^{24}$. In detail, cells were maintained in ES medium (Dulbecco's modified Eagle's medium) supplemented with $15 \%$ fetal calf serum, $0.1 \mathrm{mM} \beta$-mercaptoethanol, $2 \mathrm{mM}$ L-glutamine, $0.1 \mathrm{mM}$ non-essential amino acid, $1 \%$ of nucleoside mix $(100 \times$ stock, Millipore), $1,000 \mathrm{U} \mathrm{ml}^{-1}$ recombinant leukaemia inhibitory factor (Chemicon) and $50 \mathrm{U} \mathrm{ml}^{-1}$ penicillin/streptomycin).}

ChIP-seq. ChIP assays were performed as described previously ${ }^{24}$. Flag-bio tagged ES cells (Supplementary Datasets 1,2) were fixed in $1 \%$ formaldehyde for $7 \mathrm{~min}$ at room temperature. The formaldehyde was quenched using final $125 \mathrm{mM}$ glycine before harvesting cells. Sonicated chromatin extracts containing DNA fragments were immunoprecipitated using streptavidin-conjugated magnetic beads (Dynabeads MyOne Streptavidin T1). After washing and reverse crosslinking, purified ChIP DNA was applied for generation of sequencing libraries.

Published ChIP-seq data analysis. ChIP-seq data from mouse ES cells published before 19 Dec 2012 were downloaded from Sequence Read Archive (SRA) in National Center for Biotechnology Information (NCBI) database. Downloaded data are listed in Supplementary Dataset 1 (DNA-binding proteins) and Supplementary Dataset 3 (chromatin status). FASTQ files were extracted with the SRA Toolkit version 2.1.6 and aligned using Bowtie 2.1.0 (ref. 50) onto the mouse genome (mm9, NCBI Build 37). For the identification of DBP-binding sites (Supplementary Dataset 1), model-based analysis for ChIP-seq peak caller (MACS
1.4.2; ref. 51) was used with a dynamic local lambda calculation and building shifting model with a $P$ value cutoff of $1 \mathrm{e}-5$. Regions containing specific histone marks (Supplementary Dataset 3) were identified with random Poisson distribution without a local lambda calculation or building the peak shape shifting model. For each peak calling, author-provided control ChIP-seq data were used to remove the background noise. For the experiments performed without any control ChIP reactions, sequencing data from whole-cell extract (GSM307154) were used as a universal control.

For the multiple ChIP-seq experiments for a single DBP (Supplementary Datasets 1,6), histone modification or DNase I hypersensitivity (Supplementary Dataset 3), ChIP-seq experiments carried out at different laboratories were treated as biological replicates and those from the same laboratory were treated as technical replicates. For technical replicates, only intersection regions of peaks from all replicates were used. On the other hand, for biological replicates, consensus peak regions from at least two experiments performed in different laboratories were used for the subsequent peak based analyses or identifications of DBP co-occupied regions (Fig. 1c ( $y$ axis), Fig. 1d ( $y$ axis), Fig. 3a (MYC and PRC class cooccupancies), Figs $3 c$ and $5 a$ (Core class co-occupancy), Figs 5a-d ( $x$ axis), Fig. 6a ( $y$ axis) and Supplementary Figs 1b,c, 3a,b and 5a,b ( $x$ axis)).

Filtering low-quality ChIP-seq data. To monitor the quality of DBP ChIP-seq data, a signal-to-noise ratio (SNR) was calculated from duplicate read filtered bedGraph files generated by MACS for each ChIP-seq data as follows:

$\mathrm{SNR}=$ [area under signal curve within peak regions]/[area under signal curve outside of peak regions].

After filtering out all low-quality data with a stringent filtering criterion of $>0.015$ SNR, a total of 105 high-quality DBP ChIP-seq data out of 166 tested were used for the further analyses (Supplementary Dataset 2).

CpG methylation analysis. For the mouse ES cells CpG methylation data used in Fig. 1c,d, bisulfite sequencing data from GSM1127953 (ref. 52) was used. In order to evaluate methylation status, FASTQ files were aligned with Bismark 0.10 .0 and methylation was monitored with methylation extractor software ${ }^{53}$. Overall CpG methylation status in DBP peaks (Fig. 1c) or DBP co-occupied regions (Fig. 1d) were calculated by averaging methylation portions of all called CpG sequence within given regions.

Comparison of DBP co-occupancy. The degree of co-occupancy between two DBPs shown in Fig. 1a was measured with the deviation of observed co-occupancy from the expected values determined by randomization. In detail, the binding sites of a DBP were randomly shuffled for 1,000 times with shuffleBed software in BEDtools suite v2.17.0 (ref. 54). In order to avoid bias arising from unmappable repetitive sequences, shuffling was performed only within the genomic regions occupied by at least one DBP. Moreover, to minimize the noise derived from the sex determining chromosome (chrY) used in ChIP-seq experiments, only the peaks in the $\mathrm{X}$ and somatic chromosomes were used. For each randomization, the length distribution of each DBP co-occupied region was monitored, and the $Z$-score representing the extent of co-occupancy was calculated from the mean and s.d. of expected length of co-occupied regions.

Annotation of multiple DBP target loci and associated chromatin modification marks. For the identification of DBP co-occupied regions, DBP-bound regions within a class defined in Fig. la were merged using mergeBed software in BEDtools suite v2.17.0 (ref. 54). In order to examine the association of these DBP co-occupied regions with chromatin modification (Fig. 1d), histone marks were examined within the 300 -bp regions from the centre of the merged peaks.

Classification of promoters with CGI. CGI promoters were defined as regions containing any pre-defined CGI elements within a \pm 500 -bp region from the TSSs of all annotated genes. In order to minimize false annotations, two CGI lists, determined by independent methods ${ }^{18,55}$ were used to map consensus CGI + or CGI - promoter. In case of the gene containing multiple TSSs, only a single TSS showing the highest expression value in RNA-seq (GSM1005490; ref. 56) was used for the further analysis, to minimize the noise from rarely expressed minor transcripts.

RNA-seq analysis. RNA-seq data from mouse ES cells (GSM1005490; ref. 56) were downloaded from SRA. FASTQ files were aligned to the mouse genome (mm9, NCBI Build 37) using TopHat ${ }^{57}$. For the ENCODE RNA-seq data sets ${ }^{58,59}$ (ES cells, adult liver, B cell and $\mathrm{C} 2 \mathrm{C} 12$ in Fig. 6c), aligned bam files were downloaded and used. Gene expression was calculated as reads per kilobase per million (RPKM; single-end sequencing data, GSM1005490) or fragments per kilobase per million (FPKM; paired-end sequencing data, ENCODE) values using Cufflinks ${ }^{60}$. As ranges of RPKM values span over three orders of magnitude and tend to give high random multiplicative error in high expression values, expression values were converted into $\log _{10}$ scale $\left(\log _{10}(\mathrm{RPKM}+1)\right)$ to collapse the original range for graphical summarization. 
Microarray analysis. As a unified gene expression profile of diverse tissues and cell lines shown in Figs 2c and 4, microarray data from GNF (Genomics Institute of the Novartis Research Foundation) Mouse Gene Atlas V3 (GSE10246; ref. 34) were used. For the precise monitoring of expression values, raw data files (.cel files) were background-corrected and normalized with Robust Multi-Array expression measure using sequence information (GCRMA) ${ }^{61}$ methods to minimize the background signal originate from probe sequence or high GC contents. For genes with multiple probesets, only probes with maximal signal were used for the further analyses. For the clustering analysis shown in Fig. 4a,b, the average expression value from biological replicates was calculated in a natural scale, and each expression value was converted into a $Z$-score and clustered by unsupervised hierarchical clustering method using Xcluster software (http://www.stanford.edu/ group/sherlocklab/cluster.html). For the expression data upon knock out of the Oct4 gene shown in Fig. 6b and Supplementary Fig. 7, the microarray data set from GSE10477 (ref. 62) was downloaded and normalized with the Robust Multi-array Average $^{63}$ method. For the expression data upon induction of MYOD in mouse embryonic fibroblast cell in Fig. 6d, the microarray data set from GSE6487 (ref. 64) was used

Signal density normalization of ChIP-seq profiling. The read density of each ChIP-seq data was normalized to show the data in the same scale. For each ChIPseq data, total area under the signal curve from duplicate read filtered bedGraph files generated by MACS was considered as one billion $\left(1 \times 10^{9}\right)$. As a result, normalized signal density was shown as signal per billion as follows:

Normalized signal density $=$ area under signal curve within region $\times 10^{9} /$ total area under signal curve

Representative ChIP-seq density profile analysis. For the ChIP-seq density profile analyses (Fig. 3a (excluding MYC and PRC class co-occupancies), Figs $3 \mathrm{~b}$ and $5 \mathrm{a}$ (excluding Core class co-occupancy), Fig. 5d ( $y$ axis), Fig. 5e and Supplementary Figs 4a,c and 5a ( $y$ axis)), the following ChIP-seq data listed below were used as the representative one: H3 (GSM594580), H3K4me3 (GSM590111), H3K27me3 (GSM747539), H3K27ac (GSM851278), H3K4mel (GSM845243), Pol II (GSM632040), OCT4 (GSM307137), NANOG (GSM307140), SOX2 (GSM288347), P300 (GSM723018), LSD1 (GSM637282), CHD7 (GSM558674), MED1 (GSM560348) and MED12 (GSM560345).

Calculation of DBP-binding enrichment. In order to calculate the DBP-binding enrichment of a gene, $\log _{10}$ ratio of normalized tag density from a DBP ChIP-seq over the control ChIP-seq was used as follows:

DBP-binding enrichment $=\log _{10}\{($ normalized DBP ChIP signal in area +1$) /$ (normalized control ChIP signal in area +1$)\}$.

For the MYC and PRC class enrichment shown in Fig. 3b, median enrichment values among $18 \mathrm{MYC}$ class DBPs and 5 PRC class DBPs (excluding TET1 and KDM2A from the PRC class in Fig. 1a) were used, respectively.

TSS state mapping. Based on the existence ${ }^{19}$ (Supplementary Fig. $3 b$ ) of $\mathrm{H} 3 \mathrm{~K} 4 \mathrm{me} 3$ and $\mathrm{H} 3 \mathrm{~K} 27 \mathrm{me} 3$ marks within a $1 \mathrm{~kb}$ ( $\pm 500 \mathrm{bp}$ ) region surrounding the TSSs, the promoters of all protein-coding genes were classified into four classes of histone status as follows; active (H3K4me3 $\left.{ }^{+}, \mathrm{H} 3 \mathrm{~K} 27 \mathrm{me} 3^{-}\right)$, bivalent $\left(\mathrm{H} 3 \mathrm{~K}_{4} \mathrm{me} 3^{+}, \mathrm{H} 3 \mathrm{~K} 27 \mathrm{me} 3^{+}\right)$, repressive $\left(\mathrm{H} 3 \mathrm{~K} 4 \mathrm{me} 3^{-}, \mathrm{H} 3 \mathrm{~K} 27 \mathrm{me} 3^{+}\right)$and nonmarked (H3K4me3 ${ }^{-}$and $\mathrm{H} 3 \mathrm{~K} 27 \mathrm{me} 3^{-}$).

Gene set overlap test. The list of expression verified genes during development (Fig. 4c) was downloaded from Gene Expression Database on the MGI website (http://www.informatics.jax.org/expression.shtml ${ }^{39}$. Functional annotation of genes (GOBP; Supplementary Fig. 6a) was downloaded from the Gene Ontology website (http://www.geneontology.org/) ${ }^{65}$. Genes involved in embryonic lethality (Fig. 4d) upon mutation and gene-phenotype relations (Supplementary Fig. 6b) were extracted from genotypes and mammalian phenotype annotations on the MGI website ${ }^{40}$. Tissue-specific gene lists (Fig. 4e) were downloaded from the Unigene website (http://www.ncbi.nlm.nih.gov/unigene). Gene set overlap tests were performed with hypergeometric distribution analysis using software R (http:// www.r-project.org/). For multiple testing correction, hypergeometric probabilities were corrected by Benjamini and Hochberg ${ }^{66}$ false discovery rate. When the overlaps between two gene sets were overrepresented or underrepresented compared with expectations, they were considered as enriched or depleted, respectively.

Definition of previously defined enhancers. Active and poised (Supplementary Fig. 5a,b) enhancers were determined as previously described ${ }^{43}$. In detail, P300 peaks containing $\mathrm{H} 3 \mathrm{~K} 4 \mathrm{me} 1$, but not $\mathrm{H} 3 \mathrm{~K} 4 \mathrm{me} 3$ marks, were divided into two groups based on the presence of $\mathrm{H} 3 \mathrm{~K} 27 \mathrm{me} 3$. Previously identified active and poised enhancers ${ }^{46}$, as well as Enh elements ${ }^{45}$, were downloaded from the Supplementary Data provided by the authors; their genomic coordinates were converted into the mouse genome ( $\mathrm{mm} 9$, NCBI Build 37) using Liftover software (http://genome.ucsc.edu/util.html). ChromHMM ${ }^{47}$ enhancer elements were identified by running ChromHMM software with 10 histone modifications and chromatin accessibility defined in Fig. 1c (excluding me-CpG).

Mapping of enhancer/DBP target genes. To map the target genes of DBPs or enhancers, genes that are occupied by DBPs or enhancer (Supplementary Dataset 4) within $\pm 5 \mathrm{~kb}$ from their TSSs were considered as the target genes (Figs $5 \mathrm{c}$ and $6 \mathrm{a}-\mathrm{e}$ ). For the mapping of gene that are regulated by multiple enhancers (Fig. 5f), genes containing both proximal ( $\pm 1 \mathrm{~kb}$ from TSSs) and distal $( \pm 20 \mathrm{~kb}$ from TSSs) enhancers were used. Genes directly interacting with enhancers or OCT4-bound regions (Fig. 5f and Supplementary Fig. 7) were mapped using RNA pol II chromatin interaction analysis with paired-end tagging data from GSM1084137 (ref. 48).

Data sets for tissue-specific enhancer binding DBPs. Target loci of tissue specific DBPs in Fig. 6c were identified using following data. OCT4 (GSM288354 ref. 13), FOXA2 (GSM717562 and GSM717563; ref. 67), PU.1 (GSM537989; ref. 68) and MYOD (SRP001761; ref. 69).

\section{References}

1. Blackwood, E. M. \& Kadonaga, J. T. Going the distance: a current view of enhancer action. Science 281, 60-63 (1998).

2. Kadonaga, J. T. Regulation of RNA polymerase II transcription by sequencespecific DNA binding factors. Cell 116, 247-257 (2004).

3. Strahl, B. D. \& Allis, C. D. The language of covalent histone modifications. Nature 403, 41-45 (2000).

4. Berger, S. L. The complex language of chromatin regulation during transcription. Nature 447, 407-412 (2007).

5. Ptashne, M. How eukaryotic transcriptional activators work. Nature 335, 683-689 (1988).

6. Johnson, D. S., Mortazavi, A., Myers, R. M. \& Wold, B. Genome-wide mapping of in vivo protein-DNA interactions. Science 316, 1497-1502 (2007).

7. Boyer, L. A. et al. Core transcriptional regulatory circuitry in human embryonic stem cells. Cell 122, 947-956 (2005).

8. Mitsui, K. et al. The homeoprotein Nanog is required for maintenance of pluripotency in mouse epiblast and ES cells. Cell 113, 631-642 (2003).

9. Chambers, I. et al. Functional expression cloning of Nanog, a pluripotency sustaining factor in embryonic stem cells. Cell 113, 643-655 (2003).

10. Nichols, J. et al. Formation of pluripotent stem cells in the mammalian embryo depends on the POU transcription factor Oct4. Cell 95, 379-391 (1998).

11. Yuan, H., Corbi, N., Basilico, C. \& Dailey, L. Developmental-specific activity of the FGF-4 enhancer requires the synergistic action of Sox2 and Oct-3. Genes Dev. 9, 2635-2645 (1995).

12. Kim, J. et al. A Myc network accounts for similarities between embryonic stem and cancer cell transcription programs. Cell 143, 313-324 (2010).

13. Chen, X. et al. Integration of external signaling pathways with the core transcriptional network in embryonic stem cells. Cell 133, 1106-1117 (2008).

14. Blackledge, N. P. \& Klose, R. CpG island chromatin: a platform for gene regulation. Epigenetics 6, 147-152 (2011).

15. Deaton, A. M. \& Bird, A. CpG islands and the regulation of transcription. Genes Dev. 25, 1010-1022 (2011)

16. Zhu, J., He, F., Hu, S. \& Yu, J. On the nature of human housekeeping genes. Trends Genet. 24, 481-484 (2008).

17. Larsen, F., Gundersen, G., Lopez, R. \& Prydz, H. CpG islands as gene markers in the human genome. Genomics 13, 1095-1107 (1992).

18. Gardiner-Garden, M. \& Frommer, M. CpG islands in vertebrate genomes. J. Mol. Biol. 196, 261-282 (1987)

19. Bernstein, B. E. et al. A bivalent chromatin structure marks key developmental genes in embryonic stem cells. Cell 125, 315-326 (2006).

20. Mikkelsen, T. S. et al. Genome-wide maps of chromatin state in pluripotent and lineage-committed cells. Nature 448, 553-560 (2007)

21. Blackledge, N. P. et al. CpG islands recruit a histone H3 lysine 36 demethylase. Mol. Cell 38, 179-190 (2010)

22. $\mathrm{Wu}, \mathrm{H}$. et al. Dual functions of Tet1 in transcriptional regulation in mouse embryonic stem cells. Nature 473, 389-393 (2011)

23. Clouaire, T. et al. Cfp1 integrates both CpG content and gene activity for accurate H3K4me3 deposition in embryonic stem cells. Genes Dev. 26, 1714-1728 (2012).

24. Kim, J., Chu, J., Shen, X., Wang, J. \& Orkin, S. H. An extended transcriptional network for pluripotency of embryonic stem cells. Cell 132, 1049-1061 (2008).

25. Rahl, P. B. et al. c-Myc regulates transcriptional pause release. Cell 141, 432-445 (2010).

26. Whyte, W. A. et al. Master transcription factors and mediator establish superenhancers at key cell identity genes. Cell 153, 307-319 (2013).

27. Kagey, M. H. et al. Mediator and cohesin connect gene expression and chromatin architecture. Nature 467, 430-435 (2010).

28. Lin, C. et al. Dynamic transcriptional events in embryonic stem cells mediated by the super elongation complex (SEC). Genes Dev. 25, 1486-1498 (2011). 
29. $\mathrm{Ku}, \mathrm{M}$. et al. Genomewide analysis of PRC1 and PRC2 occupancy identifies two classes of bivalent domains. PLoS Genet. 4, e1000242 (2008).

30. Guccione, E. et al. Myc-binding-site recognition in the human genome is determined by chromatin context. Nat. Cell. Biol. 8, 764-770 (2006).

31. Heintzman, N. D. et al. Distinct and predictive chromatin signatures of transcriptional promoters and enhancers in the human genome. Nat. Genet. 39, 311-318 (2007)

32. Terranova, R. et al. Polycomb group proteins Ezh2 and Rnf2 direct genomic contraction and imprinted repression in early mouse embryos. Dev. Cell 15, 668-679 (2008)

33. Francis, N. J., Kingston, R. E. \& Woodcock, C. L. Chromatin compaction by a polycomb group protein complex. Science 306, 1574-1577 (2004).

34. Lattin, J. E. et al. Expression analysis of G Protein-Coupled Receptors in mouse macrophages. Immunome. Res. 4, 5 (2008).

35. Nie, Z. et al. c-Myc is a universal amplifier of expressed genes in lymphocytes and embryonic stem cells. Cell 151, 68-79 (2012).

36. Lin, C. Y. et al. Transcriptional amplification in tumor cells with elevated c-Myc. Cell 151, 56-67 (2012).

37. Lehmann, L. et al. Polycomb repressive complex 1 (PRC1) disassembles RNA polymerase II preinitiation complexes. J. Biol. Chem. 287, 35784-35794 (2012).

38. Roider, H. G., Lenhard, B., Kanhere, A., Haas, S. A. \& Vingron, M. CpGdepleted promoters harbor tissue-specific transcription factor binding signalsimplications for motif overrepresentation analyses. Nucleic Acids Res. 37, 6305-6315 (2009).

39. Finger, J. H. et al. The mouse Gene Expression Database (GXD): 2011 update. Nucleic Acids Res. 39, D835-D841 (2011).

40. Eppig, J. T., Blake, J. A., Bult, C. J., Kadin, J. A. \& Richardson, J. E. The Mouse Genome Database (MGD): comprehensive resource for genetics and genomics of the laboratory mouse. Nucleic Acids Res. 40, D881-D886 (2012).

41. Buecker, C. \& Wysocka, J. Enhancers as information integration hubs in development: lessons from genomics. Trends Genet. 28, 276-284 (2012).

42. Schnetz, M. P. et al. CHD7 targets active gene enhancer elements to modulate ES cell-specific gene expression. PLoS Genet. 6, el001023 (2010).

43. Rada-Iglesias, A. et al. A unique chromatin signature uncovers early developmental enhancers in humans. Nature 470, 279-283 (2011).

44. Rada-Iglesias, A. et al. Epigenomic annotation of enhancers predicts transcriptional regulators of human neural crest. Cell Stem Cell 11, 633-648 (2012).

45. Chen, C. Y., Morris, Q. \& Mitchell, J. A. Enhancer identification in mouse embryonic stem cells using integrative modeling of chromatin and genomic features. BMC Genomics 13, 152 (2012).

46. Creyghton, M. P. et al. Histone H3K27ac separates active from poised enhancers and predicts developmental state. Proc. Natl Acad. Sci. USA 107, 21931-21936 (2010).

47. Ernst, J. \& Kellis, M. ChromHMM: automating chromatin-state discovery and characterization. Nat. Methods 9, 215-216 (2012).

48. Zhang, Y. et al. Chromatin connectivity maps reveal dynamic promoterenhancer long-range associations. Nature 504, 306-310 (2013).

49. Ong, C.-T. \& Corces, V. G. Enhancer function: new insights into the regulation of tissue-specific gene expression. Nat. Rev. Genet. 12, 283-293 (2011).

50. Langmead, B., Trapnell, C., Pop, M. \& Salzberg, S. Ultrafast and memoryefficient alignment of short DNA sequences to the human genome. Genome Biol. 10, R25 (2009).

51. Zhang, Y. et al. Model-based analysis of ChIP-seq (MACS). Genome Biol. 9, R137 (2008).

52. Habibi, E. et al. Whole-genome bisulfite sequencing of two distinct interconvertible DNA methylomes of mouse embryonic stem cells. Cell Stem Cell 13, 360-369 (2013).

53. Krueger, F. \& Andrews, S. R. Bismark: a flexible aligner and methylation caller for Bisulfite-Seq applications. Bioinformatics 27, 1571-1572 (2011).

54. Quinlan, A. R. \& Hall, I. M. BEDTools: a flexible suite of utilities for comparing genomic features. Bioinformatics 26, 841-842 (2010).

55. Wu, H., Caffo, B., Jaffee, H. A., Irizarry, R. A. \& Feinberg, A. P. Redefining CpG islands using hidden Markov models. Biostatistics 11, 499-514 (2010).

56. Teif, V. B. et al. Genome-wide nucleosome positioning during embryonic stem cell development. Nat. Struct. Mol. Biol. 19, 1185-1192 (2012).

57. Kim, D. et al. TopHat2: accurate alignment of transcriptomes in the presence of insertions, deletions and gene fusions. Genome Biol. 14, R36 (2013).
58. ENCODE. A user's guide to the encyclopedia of DNA elements (ENCODE). PLoS Biol. 9, e1001046 (2011).

59. Stamatoyannopoulos, J. A. et al. An encyclopedia of mouse DNA elements (Mouse ENCODE). Genome Biol. 13, 418 (2012).

60. Trapnell, C. et al. Transcript assembly and quantification by RNA-Seq reveals unannotated transcripts and isoform switching during cell differentiation. Nat Biotechnol. 28, 511-515 (2010).

61. Wu, Z., Irizarry, R. A., Gentleman, R., Martinez-Murillo, F. \& Spencer, F. A model-based background adjustment for oligonucleotide expression arrays. J. Am. Stat. Assoc. 99, 909-917 (2004).

62. Endoh, M. et al. Polycomb group proteins Ring1A/B are functionally linked to the core transcriptional regulatory circuitry to maintain ES cell identity. Development 135, 1513-1524 (2008)

63. Irizarry, R. A. et al. Exploration, normalization, and summaries of high density oligonucleotide array probe level data. Biostatistics 4, 249-264 (2003)

64. Di Padova, M., Caretti, G., Zhao, P., Hoffman, E. P. \& Sartorelli, V. MyoD acetylation influences temporal patterns of skeletal muscle gene expression. J. Biol. Chem. 282, 37650-37659 (2007).

65. Ashburner, M. et al. Gene ontology: tool for the unification of biology. Nat. Genet. 25, 25-29 (2000).

66. Benjamini, Y. \& Hochberg, Y. Controlling the false discovery rate: a practical and powerful approach to multiple testing. J. R. Stat. Soc. Ser. B 57, 289-300 (1995).

67. Li, Z., Schug, J., Tuteja, G., White, P. \& Kaestner, K. H. The nucleosome map of the mammalian liver. Nat. Struct. Mol. Biol. 18, 742-746 (2011).

68. Heinz, S. et al. Simple combinations of lineage-determining transcription factors prime cis-regulatory elements required for macrophage and B cell identities. Mol. Cell 38, 576-589 (2010).

69. Cao, Y. et al. Genome-wide MyoD binding in skeletal muscle cells: a potential for broad cellular reprogramming. Dev. Cell. 18, 662-674 (2010).

\section{Acknowledgements}

We thank Drs Stuart Orkin, Jian Xu, Angel Syrett and Ms Laurie Cannon and Kendra Cook for their critical reading of the manuscript, and the Genome Sequencing and Analysis Facility at UT Austin for ChIP sample processing. We acknowledge the Texas Advanced Computing Center for providing HPC (high performance computing) resources. The project is supported by awards R00GM088384 from the National Institutes of Health/National Institute of General Medical Sciences (NIH/NIGMS), and R1106 from the Cancer Prevention Research Institute of Texas (CPRIT) to J.K. J.K. is a CPRIT scholar.

\section{Author contributions}

S.B., B.K.L., A.J.W. and J.K. conceived of the study; B.K.L. and C.R. performed ChIP-sec experiments; S.B., B.K.L. and J.S. analysed the data; and S.B., B.K.L., J.S., A.J.W. and J.K wrote the manuscript.

\section{Additional information}

Accession codes. bioChIP-seq data of NANOG, DAX1, MYC, MAX, E2F4, TIP60, and DMAP1 have been submitted to Gene Expression Omnibus (GEO) under accession number GSE48666.

Supplementary Information accompanies this paper at http://www.nature.com/ naturecommunication

Competing financial interests: The authors declare no competing financial interests.

Reprints and permission information is available online at http://npg.nature.com/ reprintsandpermissions/

How to cite this article: Beck, S. et al. CpG island-mediated global gene regulatory modes in mouse embryonic stem cells. Nat. Commun. 5:5490 doi: 10.1038/ncomms6490 (2014)

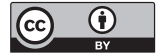

This work is licensed under a Creative Commons Attribution 4.0 International License. The images or other third party material in this article are included in the article's Creative Commons license, unless indicated otherwise in the credit line; if the material is not included under the Creative Commons license, users will need to obtain permission from the license holder to reproduce the material. To view a copy of this license, visit http://creativecommons.org/licenses/by/4.0/ 